\title{
CONTROL DE CALIDAD ÓPTICO DE GEM FOILS
}

\author{
OPTICAL QUALITY CONTROL OF GAS \\ ELECTRONMULTIPLIER FOILS
}

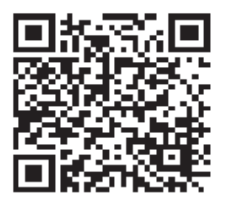

César A. Rodríguez ${ }^{1}$, Rafael M. Gutiérrez ${ }^{1}$, Andrés E. Jaramillo ${ }^{1}$ ${ }^{1 .}$ Grupo de Sistemas Complejos Universidad Antonio Nariño Bogotá, Colombia Recibido: 10 Febrero de 2015

Aceptado: 8 Marzo de 2016

*Correspondencia del autor: César A. Rodríguez. E-mail: cesararodriguez@uan.edu.co

\begin{abstract}
RESUMEN
El Multiplicador de Electrones de detectores Gaseosos (GEM) es una de las tecnologías útiles y potentes de detectores gaseosos de ionización usados en física de altas energías, física médica y otras aplicaciones. Uno de los elementos básicos de un GEM es la placa con micro perforaciones. Las características de tales perforaciones son fundamentales para un desempeño óptimo del GEM. En este trabajo se comparan diferentes métodos computacionales (implementados en lenguaje de programación Java) con el fin de determinar la calidad de las placas-GEM a partir de imágenes de alta resolución de dichas placas. El método computacional propuesto proporciona una solución alternativa automática y de alta precisión con respecto a las técnicas manuales lentas e imprecisas que se emplean actualmente, las cuales representan una limitación para el desarrollo y aplicación de esta tecnología de detectores.
\end{abstract}

Palabras claves: placa multiplicadora, detector gaseoso, control de calidad.

\begin{abstract}
The Gas Electron Multiplier (GEM) is one of the most popular and powerful technologies for gaseous ionization detectors used in high energy physics, medical physics and other applications. One of the fundamental elements of a GEM is foils with micro perforations; the qualities of such perforations are basic for an optimal performance of the GEM. In this work we study different computational methods (implemented in Java programming language), in order to determine the quality of GEM-foils from high resolution images of the foils. This computational method will provide an automatic and high precision alternative to a procedure which, at the moment, is very expensive, slow and imprecise, being a limitation for the development and application of this important technology of detectors.
\end{abstract}

Keywords: GEM-foil, gaseous detector, optical quality control. 


\section{INTRODUCCIÓN}

El Multiplicador de Electrones para detectores Gaseosos (GEM) se introdujo en el CERN (European Organization for Nuclear Research) en 1997 (1), y desde entonces se ha usado en diferentes aplicaciones tales como física de altas energías, física médica, física nuclear y otras aplicaciones en las que interviene la interacción materia-radiación. El GEM es un tipo de detector de ionización gaseoso que permite recolectar los electrones que resultan por la radiación ionizante. Es resistente, económico y proporciona un gran desempeño, así como una gran eficiencia en la detección (sobre el $98 \%$ ) (2).

El proceso físico con el cual funcionan estos detectores corresponde a la ionización de átomos o moléculas de un gas, generada por radiación o partículas que atraviesan el gas con una energía y unas propiedades determinadas. Los electrones producto de la ionización, son llevados por un campo eléctrico de difusión a una zona de campo eléctrico intenso generado por el GEM-foil, donde se produce una "avalancha de electrones" al ser la ionización proporcional al campo eléctrico, como se puede observar en la Figura. 1.

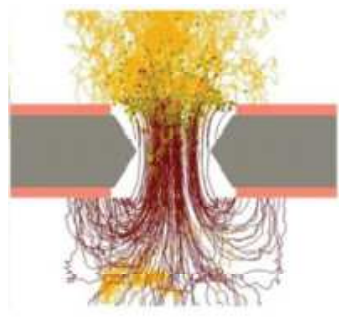

Figura 1. Simulación de una avalancha de portadores de carga en un detector GEM. (Imagen tomada del CERN Newsletter of the PH Department).

Esta amplificación permite generar una señal detectable y procesable a partir de la primera interacción que puede ser muy débil: la ionización producida por una sola partícula, por ejemplo. Esta señal es suficiente para crear una corriente que pueda ser detectada por la electrónica e interpretada por sistemas computacionales.

Considerando que la homogeneidad y control del campo eléctrico depende de la calidad y regularidad de las micro perforaciones del GEM-foil, la calidad de la información generada por este y capturada por la electrónica e interpretada por los computadores y sus interfaces, depende de la calidad de la fabricación del GEM-foils.
Un GEM-foil estándar consta de una lámina aislante de Kapton de $50 \mu \mathrm{m}$ de grosor, la cual está recubierta por ambas caras con capas de cobre de aproximadamente $5 \mu \mathrm{m}$. Esta estructura está perforada con agujeros que tienen un diámetro de $70 \mu \mathrm{m}$ cada $140 \mu \mathrm{m}$ y sus agujeros están dispuestos en un patrón hexagonal como se muestra en la figura 2(3). En el proceso de producción fotolitográfica por "etching", utilizado en la industria de los circuitos impresos y perforaciones con láser entre otros métodos que se están desarrollando, siempre se generan imprecisiones y deformaciones en la geometría y distribución de los agujeros, afectando así el rendimiento y la calidad del detector y por lo tanto del experimento: como ya se mencionó, los agujeros deformados y distribuidos irregularmente generarán diferentes propiedades del campo eléctrico y de la correspondiente amplificación, introduciendo información errónea al detector.

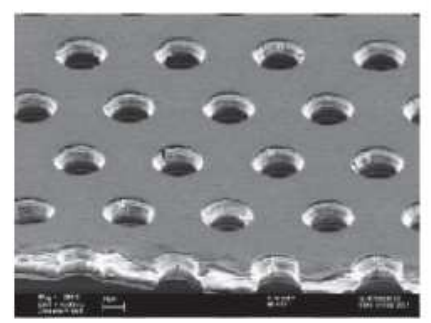

Figura 2. Imagen de la estructura de un MEG tomada con un microscopio electrónico (Imagen del CERN GDD group).

Diferentes estudios han determinado que el promedio de agujeros dañados con las técnicas de fabricación actuales, es de aproximadamente 12 por cada 500000 cubriendo un área de $100 \mathrm{~mm} \times 100 \mathrm{~mm}$, muy difíciles de detectar rápida y eficientemente considerando que es necesario revisar metros cuadrados al día con una demanda creciente, razón por la cual se necesita un método avanzado de control de calidad (4). Los resultados presentados en este trabajo se centran en la identificación de defectos que no pueden ser detectados a simple vista. Con dicho propósito se producen imágenes de alta resolución y se desarrollan programas de análisis de imágenes especializados en esta necesidad particular, con la versatilidad y flexibilidad necesarias actualmente y aquellas que se irán generando con el desarrollo de los detectores basados en MPGD's y sus crecientes aplicaciones.

El programa desarrollado en este trabajo analiza una serie de imágenes tomadas en alta resolución suministradas por el GSI de Alemania: 3488 x 2616 pixeles con un tamaño de pixel de $1,7 \mu \mathrm{m} \times 1,7 \mu \mathrm{m}$ para un 
área total aproximada de $0.6 \mathrm{~cm} \times 0.45 \mathrm{~cm}$ ) [Figura. $3]$.

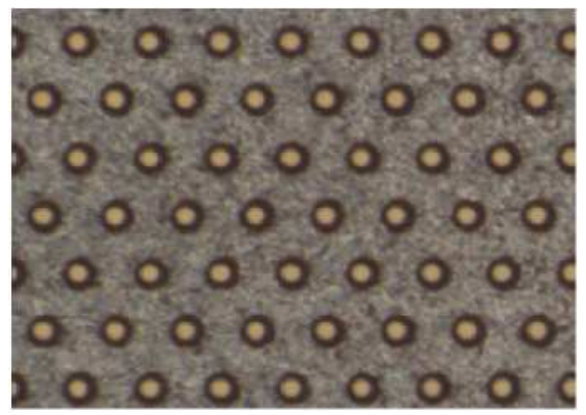

Figura. 3. Sección de imagen de una placa-MEG (Imagen tomada por el Helsinki Institute of Physics de Finlandia y suministrada por el GSI de Alemania).

La presente propuesta contribuye entonces al desarrollo de procesos de control de calidad de los GEMFoils con métodos ópticos, y fue desarrollada mediante la aplicación de métodos de identificación de formas y contornos activos con previa identificación de centros, como lo indica el siguiente esquema [Figura. 4].

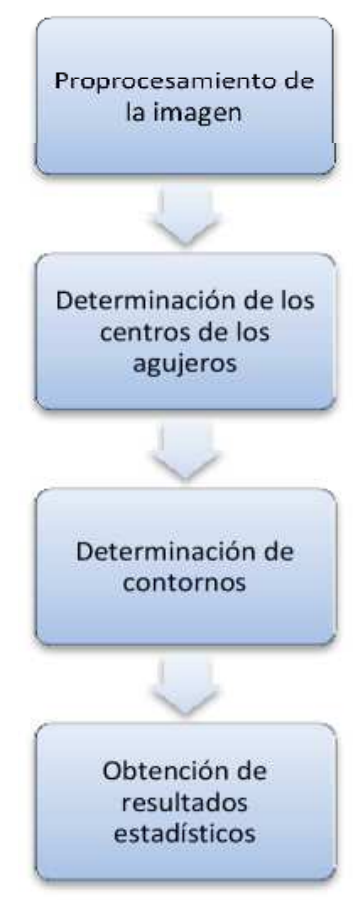

Figura 4. Diagrama de flujo del método propuesto.

En la sección 2 se presentan los métodos utilizados en el trabajo para encontrar los centros de los agujeros del foil. En la sección 3 se explican los procesos experimentales numéricos de comparación entre métodos. En la sección 4 se presentan los resultados y su discusión. Finalmente en la sección $\mathrm{V}$ se dan las conclusiones del trabajo.

\section{MÉTODOS}

En esta sección se describen los tres métodos implementados para realizar la determinación de los centros de los agujeros de los GEM-foils, y el proceso para aplicar el método de contornos activos a partir de los centros calculados.

\section{A. MÉTOdOS PARA EL CÁlCULO DE CEN- TROS}

\section{1) Transformada de Hough}

La transformada de Hough es una tecnica mayormente usada en vision por computador con la cual se pueden detectar curvas paramétricas como líneas, círculos y elipses entre otras, siendo su principal ventaja la tolerancia al ruido. Para detectar los agujeros de los foils, los cuales son círculares, se tiene según (5) que un patrón circular se puede representar por la Ec. 1 .

$$
\left(x-x_{0}\right)^{2}+\left(y-y_{0}\right)^{2}=r^{2},
$$

donde $x_{0}$ e $y_{0}$ son las coordenadas de los centros y $r$ es el radio del círculo.

La detección de los agujeros empieza con la selección de máximos locales en el área analizada la cual es asumida como el centro de la circunferencia, entonces si los índices lineales pertenecen al mínimo valor de pixel en la circunferencia, dicha área es considerada un agujero en la placa.

En este método los centros de los agujeros se identifican de la siguiente forma:

1. Conversión la imagen a escala de grises.

2. Binarización de la imagen en grises.

3. Obtención del negativo de la imagen binaria

4. Erosión de la imagen binaria con una estructura de $3 \times 3$.

5. Aplicación de la trasformada de Hough con un radio mínimo de 10 pixeles y un radio máximo de 20 y un máximo de círculos de 1500.

Estos resultados se muestran en la Figura.7.

\section{2) Imagen integral}

La imagen integral es una aplicación de las tablas de suma de áreas [Figura. 5]. Este concepto fue introdu- 
cido por Crown en 1984 (6), y es utilizado en el área de gráficos por computador.

La imagen integral se define como

$$
i i(x, y)=/_{x^{\prime} \# x, y \# y} i\left(x^{\prime}, y^{\prime}\right),
$$

donde $i i(x, y)$ es la imagen integral e $i(x, y)$ es la imagen de intensidad original. La imagen integral se puede calcular en un único paso usando las siguientes fórmulas de recurrencia:

$$
\begin{gathered}
s(x, y)=s(x, y-1)+i(x, y) \\
i i(x, y)=i i(x-1, y)+s(x, y),
\end{gathered}
$$

donde $s(x, y)$ es la suma acumulativa de la fila, $s(x,-$ $1)=0 ; i i(-1, y)=0$.

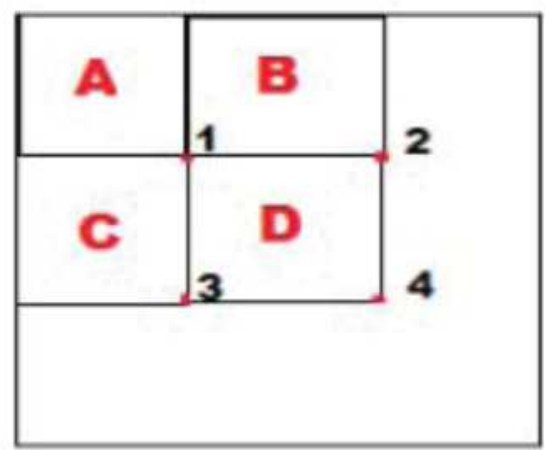

Figura 5. Imagen integral [7]

En este método los centros de los agujeros se marcaron de la siguiente forma:

1. Conversión de la imagen a escala de grises.

2. Binarización de la imagen en grises.

3. Aplicación de la Imagen integral.

4. Umbralización sobre la imagen obtenida en el paso anterior.

5. Cálculo del máximo local.

6. Aplicación de filtro de mediana.

7. Determinación de centroides.

Los centroides son los centros de los agujeros. Estos resultados se muestran en la Figura. 8.

\section{3) Convolución y máximo local}

El proceso de convolución consiste en desplazar pixel a pixel a través de toda la imagen una matriz de $H \times K$ denominada kernel, la cual es una matriz de números que se utiliza para calcular el valor del nuevo pixel a partir de sus vecinos; de lo anterior se obtiene como resultado una imagen con los valores del pixel central por donde se ha desplazado el kernel.

Según (8), la convolución de una imagen discreta se describe como la operación de una imagen $X$ de $(M$ $\times N)$ pixeles y un kernel $W$ de $(H \times K)$ coeficientes, la operación de la convolución de $X$ por $W$ se puede representar por la siguiente expresión:

$$
y(i, j)=\overbrace{h=0}^{H-1} \prod_{k=0}^{K-1} w(h, k) * x(i-h, j-k),
$$

donde $0 \leq i \leq M-1,0 \leq j \leq N-1, x(i, j)$ e $X$ y $w(\mathrm{~h}, \mathrm{j})$ e $W$.

Para este caso en particular fue usado un kernel de 27 $\times 27$ pixeles [Figura. 6], el cual permitió obtener un reforzado espacial de la imagen para luego hallar los máximos locales.

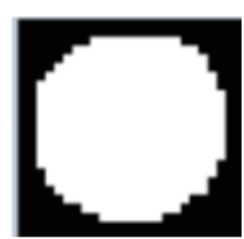

Figura 6. Kernel $27 \times 27$ pixeles

En este método los centros se identificaron de la siguiente forma:

1. Conversión la imagen a escala de grises.

2. Binarización de la imagen en grises.

3. Obtención del negativo de la imagen binaria.

4. Generación de un kernel circular de $27 \times 27$ pixeles.

5. Convolución del negativo de la imagen con el kernel.

6. Determinación de los máximos locales.

Los máximos locales corresponden a los centros de los agujeros [Figura. 9].

\section{B. CONTORNOS ACTIVOS}

Una vez encontrados los centros, la utilización de los contornos activos es fundamental para el desarrollo del presente trabajo debido a que estos permiten identificar que tan perfectos están construidos los microagujeros en el foil. Básicamente el contorno activo tiene como punto de partida los centros encontrados con los métodos propuestos y a partir de ahí éste crece como una curva paramétrica hasta los bordes de cada agujero. Los contornos activos son curvas paramétricas que pueden desplazarse desde un punto de partida hasta los bordes de un objeto o forma en una imagen 
bajo la influencia de fuerzas internas provenientes de la misma curva y fuerzas externas derivadas de la misma imagen.

Según (5), un contorno activo es una curva Spline de energía mínima cuya posición se define paramétricamente por $v(s)=(x(s), y(s))$. En el modelo básico, el contorno depende de las fuerzas internas $\left(\mathrm{E}_{\text {int }}\right)$, las cuales determinan tanto la elasticidad como la rigidez; las fuerzas de la imagen $\left(\mathrm{E}_{\text {imag }}\right)$, que tienden a rodear características tales como líneas y bordes; y fuerzas de restricción $\left(\mathrm{E}_{\mathrm{res}}\right)$ impuestas por el usuario. $\mathrm{El}$ funcional de energía viene dado por

$$
E_{\text {contorno }}=\#_{0}^{1} E_{\text {contorno }}(v(s)) d s
$$

ó

$$
E_{\text {contorno }}=\#_{0}^{1} E_{(i n t(v(s))}+\mathrm{E}_{(\operatorname{imag}(\mathrm{v}(\mathrm{s}))}+\mathrm{E}_{(\mathrm{res}(\mathrm{v}(\mathrm{s})) \mathrm{d} \mathrm{s})}
$$

Una vez aplicados los contornos activos a cada micro agujero en la imagen el programa se extrae información como el radio y tamaño, para así calcular datos como la excentricidad y luego se determina qué tan semejantes son los agujeros obtenidos respecto al modelo circular ideal (Tabla 1).

\section{RESULTADOS EXPERIMENTALES Y DIS- CUSIONES}

La técnica propuesta se implementó en lenguaje de programación Java en un computador con un procesador Intel ${ }^{\circledR}$ Core $^{\mathrm{TM}}$ i5-3570 CPU a $3.40 \mathrm{GHz}$, con 4.0 GB RAM. En el experimento se utilizaron imágenes de alta resolución tomadas por el Instituto de física de Helsinki, Finlandia y suministradas por el GSI de Alemania.

Para determinar los centros de los microagujeros se probaron tres métodos sobre una imagen con 1590 agujeros. El método de la imagen integral y el de convolución fueron más rápidos, con tiempos de $1925 \mathrm{~ms}$ y $2980 \mathrm{~ms}$, respectivamente. Para la transformada de Hough se obtuvo un tiempo de 120000 ms [Tabla 1].

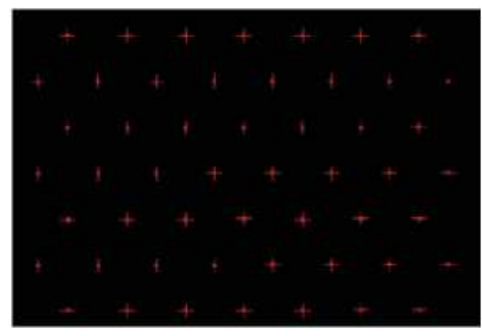

Figura 7. Centros calculados por la transformada de Hough

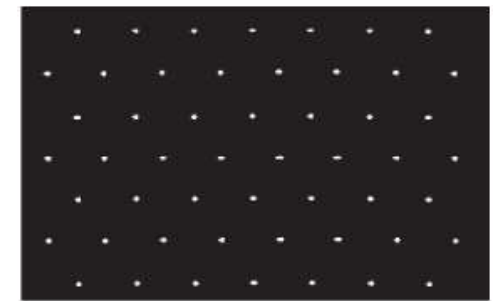

Figura 8. Centros calculados por Imagen Integral

Figura 9. Centros calculados por convolución.

De acuerdo al objetivo principal de este trabajo, que consiste en identificar los defectos en los microagujeros, se realizó una súperposición de los centros encontrados sobre la imagen original [Figura. 10], y luego se aplicó el proceso de crecimiento de los contornos activos [Figura. 11]. Parte de los resultados de este proceso se muestran en la Tabla 2.

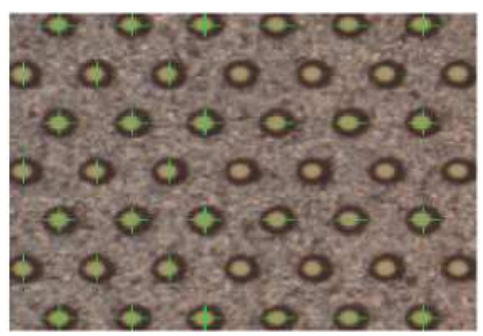

Figura 10. Centros en una imagen real: cruces verdes

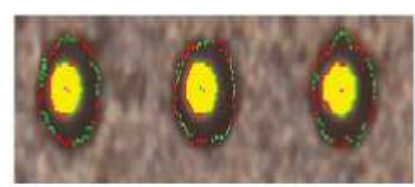

Figura 11. Contornos activos. Los contornos interno y externo se identifican en pixeles rojo y verde (sección de una imagen ampliada).

Tabla 1 Evaluación de desempeño para determinación de centros de los agujeros en las imágenes del GEM-foil

\begin{tabular}{lccc}
\hline & $\begin{array}{c}\text { A. Transf. } \\
\text { de Hough }\end{array}$ & $\begin{array}{c}\text { B. Imagen } \\
\text { Integral }\end{array}$ & $\begin{array}{c}\text { C. Convolución y } \\
\text { Máximo local }\end{array}$ \\
\hline $\begin{array}{l}\text { Tiempo de } \\
\text { proceso (ms) }\end{array}$ & 120000 & 1925 & 2980 \\
$\begin{array}{l}\text { Preprocesa- } \\
\text { miento }\end{array}$ & Bajo & Alto & Medio \\
\begin{tabular}{l} 
Falsos-Positivos \\
\hline
\end{tabular} & $0 \%$ & $0.06 \%$ & $0 \%$ \\
\hline
\end{tabular}


Tabla 2. Resultados de algunas medidas utilizadas para determinar la calidad de los GEM-foil (centros calculados por el método de convolución y máximo local).

\begin{tabular}{lcc}
\hline \multicolumn{1}{c}{ Parámetros } & Valor & Unidad \\
\hline Resolución de la imagen (.PNG) & $3488 \times 2616$ & Pixeles \\
Tamaño de archive & 21.4 & MBytes \\
Tiempo de proceso & 1743 & $\mathrm{~ms}$ \\
Agujeros Buenos & 1587 & \\
Agujeros defectuosos & 3 & \\
Radio interno (promedio) & 15,47 & $\mathrm{~mm}$ \\
Radio interno (desv. estándar) & 0,61 & $\mathrm{~mm}$ \\
Excentricidad interna (promedio) & 0,19 & \\
Excent. interna (desv. estándar) & 0,17 & \\
Aplanamiento interno horizontal & 49,3 & $\%$ \\
Aplanamiento interno vertical & 50,7 & $\%$ \\
Pitch interno 1 (promedio) & 134,35 & $\mathrm{~mm}$ \\
Pitch interno 1 (desv. estándar) & 1,05 & $\mathrm{~mm}$ \\
Pitch interno 2 (promedio) & 134,39 & $\mathrm{~mm}$ \\
Pitch interno 2 (desv. estándar) & 1,25 & $\mathrm{~mm}$ \\
\hline
\end{tabular}

\section{CONCLUSIONES}

Otros estudios han establecido que los defectos y las variaciones en las características de los microagujeros afectan el desempeño de los detectores basados en los GEM-foils (10), (11), (12). Sin embargo, aún no se han establecido rangos específicos de variación de la geometría del foil para determinar si su funcionamiento es aceptable o no. En este artículo se presenta una nueva técnica que permite obtener medidas de los agujeros de un foil a partir de las imágenes de dicha foil. Una vez establecidas las tolerancias geométricas aceptables para los agujeros los GEM-foils por parte de los fabricantes, las medidas obtenidas por este procedimiento permitirán determinar la calidad de las láminas.

Los resultados de los experimentos que se presenta en este trabajo, muestran la robustez, la precisión y la eficiencia del proceso, el cual integra aplica y desarrolla diferentes métodos para obtener resultados eficientes de alta calidad. Sin embargo, el método propuesto está en proceso de optimización.

Un reto importante a futuro es el uso de este método de caracterización por medio óptico para establecer la relación entre la calidad de los GEM-foil y el desempeño del detector, lo cual representa un avance importante en la ciencia y tecnología de los detectores. 


\section{BIBLIOGRAFÍA}

1. F. Sauli, GEM: A new concept for electron amplification in gas detectors, Nucl. Instrum. Meth. A 386, 531, 1997.

2. G. Bencivenni et al., Performance of a triple-GEM detector for high rate charged particle triggering, Nucl. Instr. and Meth. A, 494, 156, 2002

3. F. Sauli, Nucl. Instr. and Meth. A 386, pp. 53, 1997

4. M. Kalliokoski, "Study of GEM-foil defects with optical scanning system", Nuclear Science Symposium Conference Record (NSS/MIC), 2010 IEEE, pp. 1446-1449, October 2010.

5. J. Ruen, "A Circular Hough Transform Hardware for Industrial Circle Detection Applications", Industrial Electronics and Applications, 2006 1ST IEEE Conference on, pp. 1-6, May 2006.

6. T. Ephraim, "Real-Time Viola-Jones Face Detection in a Web Browser", Computer and Robot Vision, 2009. CRV '09. Canadian Conference on, May 2009.

7. J. Chatrath, "Real Time Human Face Detection Tracking", International Conference on Signal Processing and Integrated Networks, Feb 2014.

8. V.G Moshnyaga, "A new architecture for in-memory image convolution", Acoustics, Speech and Signal Processing,, IEEE International Conference. May 1998

9. D.M Kocak , "Tracking and mapping underwater bioluminescent displays using snakes", OCEANS '96. MTS/IEEE. Prospects for the 21st Century. Conference Proceedings, September 1996.

10. J. Benlloch , "Further developments and beam tests of the gas electron multiplier (GEM)," Nuclear Instruments and Methods A, vol. 419, pp. 410-417, 1998.

11. O. Bouianov, "Foil geometry effects on GEM characteristics," Nuclear Instruments and Methods A, vol. 458, pp. 698-701, 2001.

12. S. Bachmann, "Charge amplification and transfer processes in the gas electron multiplier," Nuclear Instruments and Methods A, vol. 438, pp. 376-408, 1999. 